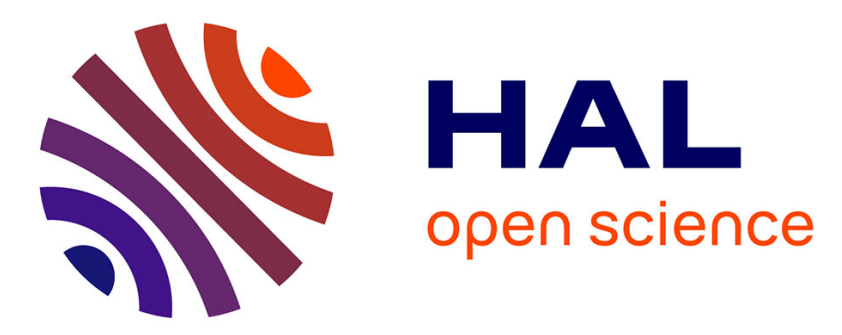

\title{
A novel Communication Technique for Nanobots based on acoustic signals
}

\author{
Valeria Loscrì, Enrico Natalizio, Valentina Mannara, Gianluca Aloi
}

\section{To cite this version:}

Valeria Loscrì, Enrico Natalizio, Valentina Mannara, Gianluca Aloi. A novel Communication Technique for Nanobots based on acoustic signals. BIONETICS, Dec 2012, Lugano, Switzerland. hal00794156

\section{HAL Id: hal-00794156 \\ https://hal.inria.fr/hal-00794156}

Submitted on 25 Feb 2013

HAL is a multi-disciplinary open access archive for the deposit and dissemination of scientific research documents, whether they are published or not. The documents may come from teaching and research institutions in France or abroad, or from public or private research centers.
L'archive ouverte pluridisciplinaire HAL, est destinée au dépôt et à la diffusion de documents scientifiques de niveau recherche, publiés ou non, émanant des établissements d'enseignement et de recherche français ou étrangers, des laboratoires publics ou privés. 


\title{
A novel Communication Technique for Nanobots based on acoustic signals
}

\author{
Valeria Loscrí ${ }^{1}$, Enrico Natalizio ${ }^{2}$ Valentina Mannara ${ }^{1}$, and Gianluca Aloi ${ }^{1}$ \\ 1 University of Calabria, Unical, 87036 Arcavacata di Rende, Italy, \\ vloscri@deis.unical.it, \\ 2 Lab. Heudiasyc - UMR CNRS 7253, Universite de Technologie \\ Compiegne, France
}

\begin{abstract}
In this work we present the simulation of a swarm of nanobots that behave in a distributed fashion and communicate through vibrations, permitting a decentralized control to treat endogenous diseases of the brain. Each nanobot is able to recognize a cancer cell, eliminate it and announces through a communication based on acoustic signals the presence of the cancer to the other nanobots. We assume that our nanodevices vibrate and these vibrations cause acoustic waves that propagate into the brain with some intensity that we evaluated by taking into account the specific physical factors of the context, the nano-metric nature of the vibrant devices and the characteristic of the fluid where the devices are immersed. An important aspect of our approach is related to the communication based on vibrations. This choice is related to the application context where is not advisable either to use indiscriminate chemical substances or electromagnetic waves. Whereas, ultrasonic waves are used in the most frequent diagnostic techniques and the use of this kind of techniques should not have negative collateral effects. Specifically, we propose an approach based on bees' behavior in order to allow our devices to communicate, coordinate and reach the common objective to destroy the cancerous tissues. In order to evaluate the effectiveness of our technique, we compared it with other techniques known in literature and simulation results showed the effectiveness of our technique both in terms of achievement of the objective, that is the destruction of the cancerous cells, and velocity of destruction.
\end{abstract}

Key words: Acoustic Communication, Nanobots, In-vivo Applications

\section{Introduction}

Nanotechnologies are a new approach based on comprehension and deep knowledge of the properties of the matter at the nanoscale level: a nanometer corresponds to the length of a small molecule. At nanoscale, behaviors and characteristics of the matter drastically change, making it necessary a synergy among many different disciplines. A great percentage of the nanosytems world is represented by technologies derived from micro-electronics, pushed up to nanoscale, in order to obtain electronic, optical, fluidic, integrated mechanic functions to 
be applied to different fields ranging from microelectronic, telecommunications and sensor, to environmental and bio-medical. The latter field could be literally revolutionized from the potential applications of nano-devices, both in the diagnostic and pharmaceutic fields [9]. One of the most interesting applications is the controlled release of drugs over time and exactly localized in cells or organs that need it, drastically reducing the side effects. Nanotechnology is already used in the field of diagnostics through the use of synthetic tracer molecules for investigating biological processes in a non-invasive fashion. In the cancer fight, as for the treatment of some diseases related to the cardiovascular system more and more often you hear speak about nano-therapy. Among the first applications to be postulated in the early '90s and also among the most fascinating of nanomedicine is the idea of using nanorobots [8]. A device of a few nanometers (nanorobots will typically be 0.5 to 3 microns large with 1-100 nm parts), could be introduced into the body without causing injury and, if equipped with sensors that transmit precise images could facilitate the early diagnosis of cancer and carry drug to the target or to perform other tasks that would otherwise require invasive surgery [1], [3]. In order to prevent attacks from immune system a nanorobot in vivo should be characterized with a smooth and flawless diamond exterior, because this prevents Ieukocytes activities since the exterior is chemically inert and have low bioactivity [2].

A nanorobot is a system able to modify the surrounding in a controlled and predictable fashion, with size at the molecular or even atomic scale. In practice, a nanorobot is either a passive or active structure able to detect, signal and elaborate information. The very limited size of the devices implies limited capabilities and reduced computation resources, therefore it is necessary to make devices collaborate by applying design techniques such as Swarm Intelligence in order to realize complex systems through the interaction and the cooperation of very simple agents [11].

This work is inspired by the paper of Lewis Anthony and Bekey George developed in 1992 at Southern California University and entitled "The Behavioral Self-Organization of Nanorobots Using Local Rules" [4], where the objective was the evaluation of a swarm of nanobots, organized without any centralized unit, only trough simple local rules, to destroy cancer cells inside the brain. Thanks to the technological development, the assumptions made in [4] are plausible and it is possible to design molecular nanobots constituted from two polymers able to deactivate the production of a protein that causes the death of the cells attached [7]. Lewis's work has an early work for the assumption of using robots for in vivo applications through a swarm technique. In [4] and in other next works [5] [6], the communications are performed by chemical mechanisms, that is, the report of either some events or object is realized through the release of chemical substances that diffuse by attracting other nanorobots through the gradient associated to the signal intensity. Specifically, Lewis's strategy is based on three chemical substances that attract the swarm of nanobots in a gradual fashion to achieve the total elimination of the diseased cells. An early part of the work is devoted to the tuning of the characteristic parameters of the algorithm where 
objective difficulties emerged, first of all the extremely reduced dimensions of the devices, their limited computational capabilities and the structural space needed to install sensors and interfaces to capture all the chemical substances involved. For these reasons, we considered a different approach inspired from bees' behaviors that use vibration to communicate the distance and the position of food sources to the rest of the colony. Our choice for this specific kind of communication has been lead by the simplicity of the components required from the devices, and the fact that many diagnostic instruments are already based on microwaves, which are considered not dangerous for the human. A useful summary of communication paradigms to interconnect nanobots in a body area network is given in [13]. Preliminary results of Bee's algorithm are presented in [15]. The rest of the paper is structured as follows. In Section II we give some details about Lewis's approach. In Section III we describe our bees's approach. In Section IV we give the details about the simulation environment and the results. Finally, we conclude the paper in Section $\mathrm{V}$ and we give some directions for future works in Section VI.

\section{Lewis Algorithm}

When Lewis and Bekey presented their work in 1992, the Swarm Intelligence concept had not yet been fully defined. In fact, authors refer to a $\mu$-colony as a set of robots that coordinate activities autonomously to perform some specific tasks. The colony could be constituted by hundreds or thousands of $\mu$-robots, and each of them has simple computational capabilities like a colony in nature. As we already outlined, the technique they proposed was based on chemical communication. Each $\mu$-robot is able to mark its surrounding through chemical substances, recognizes the different chemical signals and follows the different gradients until it reaches the cancer cells. The colony is injected close to the cancer. Once injected into the body, the $\mu$-robots move randomly until they reach cancer cells. After the first contact the $\mu$-robot emits a substance in its surrounding called CHEM-1. This substance is absorbed by the body after a certain time. A certain percentage of $\mu$-robots differentiate in guidepost, stop and start to secrete substances that permit the transmission of the signal over long distances. These substances are CHEM-2 and CHEM-3 and are used as repeaters. The number of $\mu$-robots that differentiate in guideposts determines the efficiency, which is the convergence of $\mu$-robots to defeat the tumor. The number of guideposts is very important. In fact, if too many nanobots differentiate it is difficult to reach and destroy the tumor. On the other hand, if the number of guideposts is too low, the colony is not able to complete the task. For this reason, authors considered a differentiation probability $p=0.01$ and related the total number of guideposts to the total number of $\mu$-robots $n$ and the current time $t$, pnt. In what follows we show the pseudo-code of the Lewis-Bekey approach: 
Algorithm 1 Lewis and Bekey Algorithm

IF There is no chemical Markers and No Tumor

THEN Do a random Walk

IF A Tumor is detected

THEN Destroy the cell, Differentiate and Broadcast CHEM-1; w/prob 1

IF The Mag. of CHEM-1 is greater than $\Theta$

THEN Do a random Walk

IF CHEM-1 is detected

THEN Move up the gradient of CHEM-1; w/prob $\mathrm{p}$

or differentiate and Broadcast CHEM-2; w/prob(1-p)

IF CHEM-2 is detected

THEN Move up the gradient of CHEM-2; w/prob p

or Differentiate and broadcast CHEM-3; w/prob(1-p)

IF CHEM-3 is detected

THEN Move up the gradient of CHEM-2; w/prob 1

The algorithm's efficiency is strictly related to some additional parameters, like the threshold value of CHEM-1, $\Theta$. The environment considered is the cerebral cortex that is represented in the simulator NSl [10] as a cells array. The modeled chemical communication takes into account the generation, the diffusion of the substances immersed in a fluid and the absorption factor. A single cell is the measure unit of the space and only one nanobot at a time can occupy a single cell. The cancer and the colony are positioned inside the grid. Each robot is able to move step by step in one of the 8 adjacent cells. Once a $\mu-$ robot reaches a cancer cell it is able to perceive the tumor and to signal its presence to other robots. In our simulation, we adopted two different convergence criteria. The first one is represented by the total elimination of the cancer cells and the other is a maximum number of iterations (1000), that is not an actual convergence criterion but we need to stop the simulation when the algorithm fails to eliminate all the cells. Even if the algorithm is strictly related to the setting of different parameters, the aim of the authors was mainly to show how behavioral algorithm is able to make the colony reach a common objective. It is worth to note that, the use of three chemical signals implies at least three different interfaces to capture the different substances. Moreover, a single nanobot needs to physically transport three different substances. By considering the reduced dimensions it seems very difficult to imagine how this algorithm could be realized. 


\section{Bees' Approach}

As we already outlined above, the acoustic communication we propose in this work is inspired from bees exploration technique for food searching. In practice, our nanobots borrow from the bees the capabilities to communicate through vibration (waggle dance for the bees). The self-organization of the bees is based on very simple rules related to the behavior of each individual. Moreover, the concept of swarm applied in the context of in vivo application has several advantages compared to isolated nanobot. For example, acoustical nanobots could form in vivo communication networks that could transfer data across much larger distance than possible with direct transmission by considering the attenuation at high frequencies [16]. Generally, nanorobots can improve their performance and they are able to accomplish complex tasks, by coordinating their actions in a decentrilezed fashion. A similar approach is considered in [17]. We refer to this bees' inspired technique as NanoBee and is supported by the possibility of using acoustic waves as transmission means in communications in vivo without specific risks associated to. We exploited the analysis made in [9] to characterize our simulation model and tune the parameters. The vibrations associated to the devices generate acoustic waves that propagate in an elastic medium and cause pressure variations and movement of the particles that compose the medium and that can be perceived and detected from an acoustic detector. This perturbation, that carries both the information and the energy, propagates while every particle, also in the case of a fluid, remains nearby its original position. In practice, there are local vibrations (compression and rarefaction) of the particles and in the case of fluid, where there can not be cutting efforts, the vibrations are parallel to the propagation direction of the wave, i.e. longitudinal waves. The dimension or magnitude of sound can be indifferently expressed as sound power, sound intensity or sound pressure. Sound power is the total amount of acoustic energy emitted from a sound source and is measured in watt. Sound intensity is the ratio of the power of a sound wave and the crossed superficial area, it is usually measured in $w a t t /$ meter $^{2}$. Sound pressure is the value of the pressure variation of a corps in a generic point inside the sound field and is measured in newton $/$ meter $^{2}$. It is worth to note that for a given sound, sound power is constant while both intensity and pressure depend on the specific measure conditions. The simplest case is sound or noise that propagate freely without any obstacle. With this assumption and when the medium considered is non-dissipative, intensity (I), power $(\mathrm{P})$ and pressure $(\mathrm{p})$ are correlated as it follows:

$$
I=\frac{W}{2 \pi r^{2}}=\frac{p^{2}}{\rho c}
$$

where $\rho$ is the density of the fluid and $\mathrm{c}$ is the light speed. It is worth to note that both intensity and pressure decrease with the square of the distance $(\mathrm{r})$. Based on these considerations we assume the possibility to design a technique based on acoustic communication by modeling the propagation of signals with sound intensity. NanoBee technique considers nanobots as point sources, since their size is small compared to the distance from the receiver. Each nanobot is 
able to signal the presence of a cancer cell by stopping its movement and starting vibrating, mimicking the bees behavior, in order to transmit a sound signal to alert other agents (nanobots). Every nanobot that receives the signal moves towards the gradient of the intensity received. Vibrating time is limited since it depends on the force associated to each device to accomplish the movement. In order to simulate the spatial variation of intensity sound, we assume three different probabilistic intervals, that simulate both the spatial propagation and the attenuation of the sound. Specifically, we choose higher probabilities values close to the nanobot that is dancing and probabilities values smaller when distances are greater by simulating in this way the attenuation with the square of the distance. A more realistic version of the algorithm must take into account the temporal attenuation of a sound signal which introduces a decrease of both the intensity and the distance reached by the signal. The first version, where we do not take into account the temporal attenuation of the signal is referred as NanoBee ON-OFF, where ON and OFF indicate whether the device is "ON" (it vibrates with the maximum power) otherwise it is "OFF" (and does not vibrate at all). The second version of our algorithm is referred as NanoBeeEvan and is related with a temporal attenuation of the sound signal. From a computational point of view, our devices are very simple since the algorithm only requires that each device has capabilities of:

- recognition of a cancer cell;

- destruction of a cancer cell;

- emission of vibrations to signal the position of the cancer;

- detection of acoustic waves.

In what follows we give the pseudo-code of the NanoBeeAlgorithm:

\footnotetext{
Algorithm 2 NanoBee

Repeat

for each "active" nanobot $i$ :

pick up any sound signal in its surrounding;

IF there are not any signal/cancer cells

search randomly;

IF discovered a cancer cell

THEN eliminate the cell and starts to dancing;

IF a signal has been received

THEN moves towards the higher intensity of the signal;

Until there is an "active" nanobot
}

A nanobot is active when it has enough energy, above a certain predetermined threshold and it has not discovered any cancer cell yet. 


\section{Simulation Results}

We simulate the systems previously described with Neural Simulation Language (NSL), [10]. NSL possesses many features that facilitated the simulation development, including graphics capabilities, and language constructs for handling layers of grid object. In order to evaluate our techniques in different situations we consider the possibility to have different initial configurations of the cancer. In fact, based on the specific geometry of the processes, different cancer forms characterize different cancer types. Specifically, we consider the possibility to have metastases detached from the original tissue. Usually, metastases are a group of cells and only rarely they are isolated, but for completeness we considered the three different scenarios: "aggregated", "scattered groups" and "isolated".

\subsection{Different Initial Configuration}

The space where nanobots move and act is a grid of cells modeling the vertebral cortex. In Figure 1 we show the initial configuration when the "aggregated" case is considered, in Figure 2 the initial configuration of the cancer is with "groups" of cells and in Figure 3 we show the initial configuration of the cancer with "isolated" cells. Specifically, the number of cancer cells is 42 in every configuration and the cancer cells are represented in blue, while the nanobots, that are 289 in every configuration, (as in [4]), are represented in black.

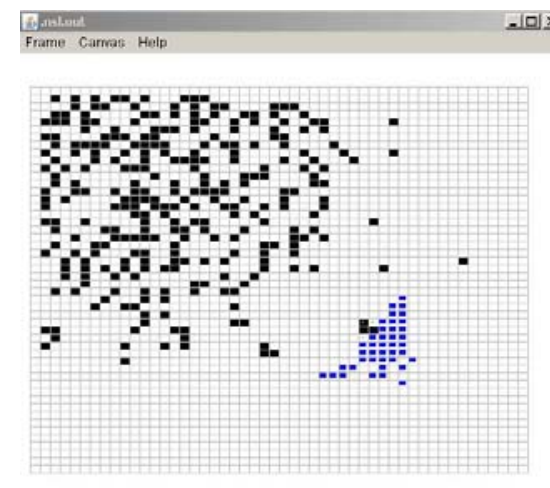

End Crete time: 57.0 Finished Crcles: 57 Finished Epochs: 0

Fig. 1. Initial Configuration of the "aggregated" case.

\subsection{Convergence Time}

The input parameters we consider in order to evaluate the performance of the various techniques are summarized in table 1 . The algorithms considered in this 


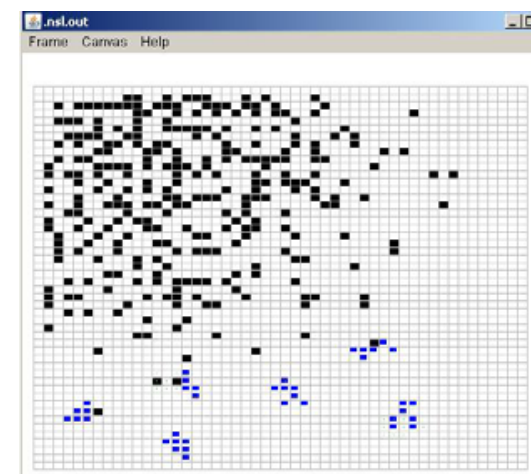

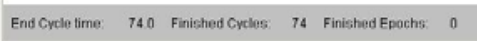

Fig. 2. Initial Configuration of the "scattered groups" case.

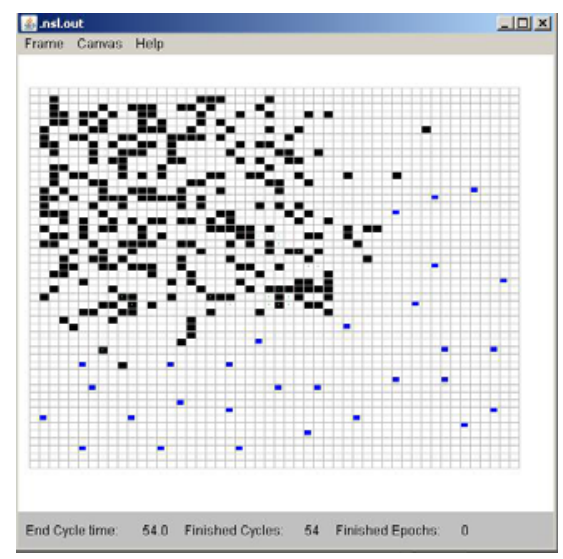

Fig. 3. Initial Configuration of the "isolated" case.

work are based on the concept of nanobots swarm, which allows us to consider a reduced dimension of devices with available power smaller than that necessary if only a device is considered as observed in [12]. Frequencies ranging from 1 to 5 $\mathrm{MHz}$ are typically used for the head [12], thus, we choose the value of frequency equal to $1 \mathrm{MHz}$. Moreover, in our bees' approach, communication activity is only related to the achievement of the target. In practice, nanobots start to vibrate when they discover a tumor and not when they move. We apply the method of Independent Replications with a confidence interval of $95 \%$.

In Fig. 4 we show the convergence time of each considered algorithm for the "aggregated" case. The first important observation is related to Lewis approach (referred in the diagram as NanoLewis) that is not able to totally destruct the cancer cells even if we consider the maximum number of nanobots. Concerning 
Table 1. Simulation Parameters

\begin{tabular}{|c||c|}
\hline Searching Space & $50 \times 50$ cells \\
\hline N Nanobots & 289 \\
\hline Cancer size & 42 cells \\
\hline Size of nanobots & $0.5 \mu \mathrm{m}$ \\
\hline Frequency & $10 \mathrm{MHz}$ \\
\hline Power & $0.5 \mathrm{pW}$ \\
\hline Simulation Time & $1000 \mathrm{runs}$ \\
\hline Prob Values & {$[1-0.7],[0.69-0.3],[0.29,0]$} \\
\hline Confidential Interval & $95 \%$ \\
\hline
\end{tabular}

Lewis'approach we had to set many parameters and after conducting a sensitivity analysis we chose the set of parameters that guarantee the best performance in terms of convergence. During the simulation we observed as the combination of the three chemical substances creates a kind of "barrier effect". In practice, after the first nanobots enters in contact with cancer cells and a part of the devices differentiate and start working as a kind of relay by sending CHEM-1. At this point, it is very difficult for the other nanobots to attack the most internal cancer cells. When the stations differentiate like "guidepost" and start send CHEM-2 if they already entered in contact with CHEM-1 or CHEM-3 if they were touched from CHEM-2, the situation worsens. Bees' approaches behave similarly, with the difference that NanoBee ON-OFF is able to eliminate all the cancer cells with less nanobots than NanoBeeEvan. NanoBee ON-OFF is more effective in this case cause of the signal propagation that reaches farther during the time and is able to attract more nanobots. NanoBeeEvan consideres the attenuation of the signal power during the time. Hence, after a certain period the signal only reaches a shorter distance.

In Fig. 5 we can observe how the NanoLewis is not yet able to destruct all the cancer cells, in the "scattered group" algorithm. In this case we observe a little improvement of the NanoBeeEvan. This latter, through the "evanescence" of the signal in the time, implies more randomness of movement of the nanobots. Hence, when tumor cells are scattered in the region of interest, this randomness is useful to reach the various points of interest more quickly.

This reasoning is confirmed in Fig. 6 where NanoBeeEvan overcomes the NanoBee ON-OFF, for the case of "isolated" cells.

It is worth to note how both the nano-bee approaches require less nanobots to reach the goal to eliminate all the cancer cells in respect of those needed by Lewis algorithm. In our opinion the analysis of the performance in terms of both effectiveness, that is the capability to destruct all the cancer cells, and the rapidity to do it by varying the number of nanobots, is very important because it is very important to use the minimum number of nanobots in a similar context. Furthermore, it is very important to try to reduce both the chemical and the acoustic messages as much as possible. 


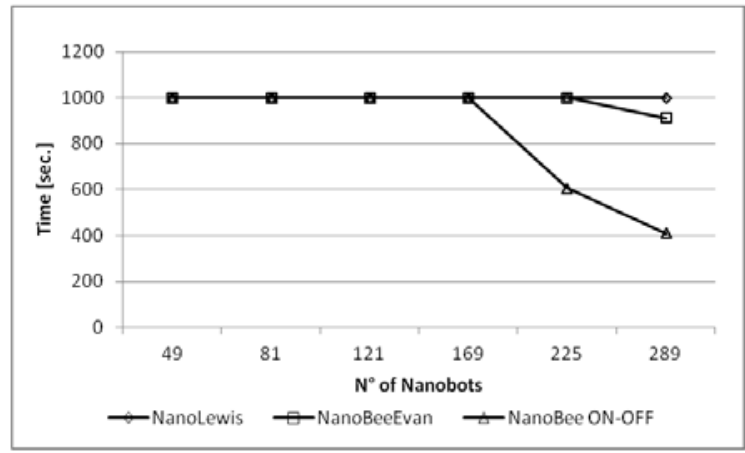

Fig. 4. Convergence Time in the case of "aggregated" configuration.

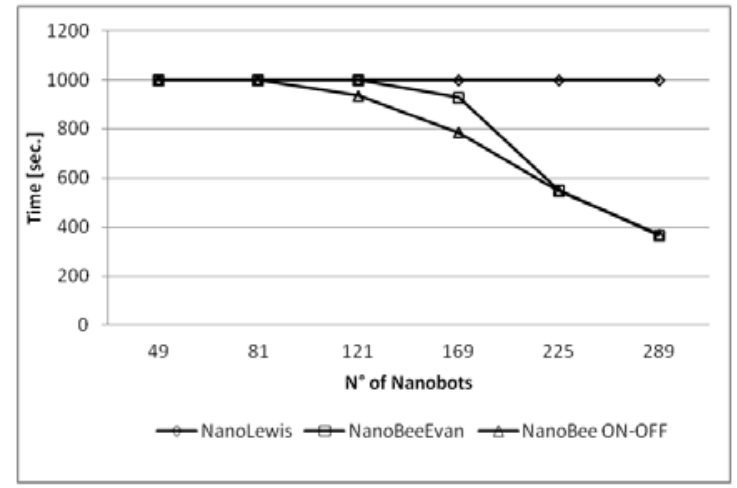

Fig. 5. Convergence Time in the case of "scattered groups" configuration.

\subsection{Amount of Eliminated Cancer Cells}

In this section we present another type of analysis related to the variation of the number of nanobots but also related to the capability to eliminate the cancer cells. The simulation parameters are always those summarized in the Table 1.

In Fig. 7 we can observe how the number of cancer cells eliminated by the NanoLewis technique is always smaller than the other two techniques. As already outlined before, we observe that the algorithm is effective when the first nanobot attaches the tumor, but the barrier created through the chemical substances does not allow to penetrate inside the body of the cancer. In fact, even if the number of nanobots increases, above all, in the first configuration (where we have only a 


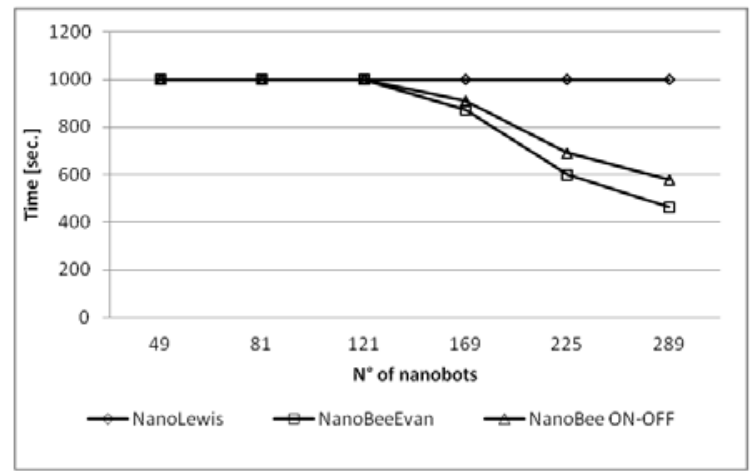

Fig. 6. Convergence Time in the case of "isolated" configuration.

single tumor), the effect does not change. This shows that the scarce efficiency is not related to the small number of nanobots. On the contrary, with our bees techniques we have that an increasing number of nanobots corresponds to an increased capability to attack the cancer. In fact, in the "aggregated" case, we pass from around 18 cells to 42 cells.

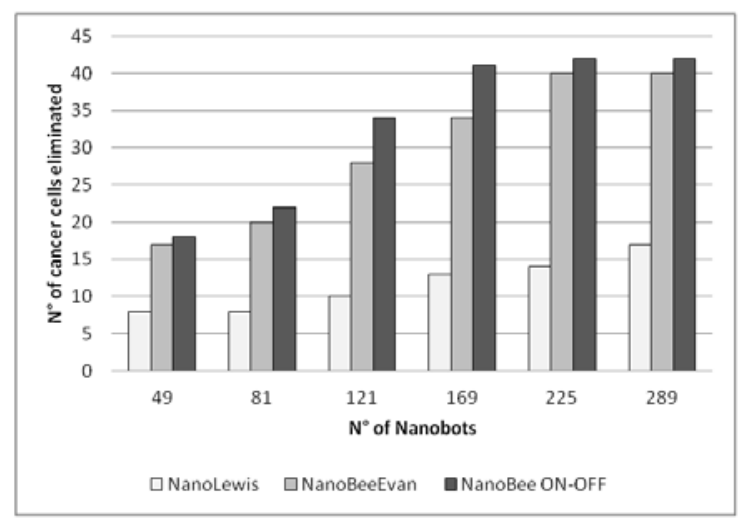

Fig. 7. Number of eliminated cancer cells in the case of "aggregated" configuration.

In Fig. 8 we can observe how the barrier effect of the NanoLewis approach is mitigated in the "scattered groups" configuration. Moreover, for this specific configuration the bees approaches behave very similarly. 


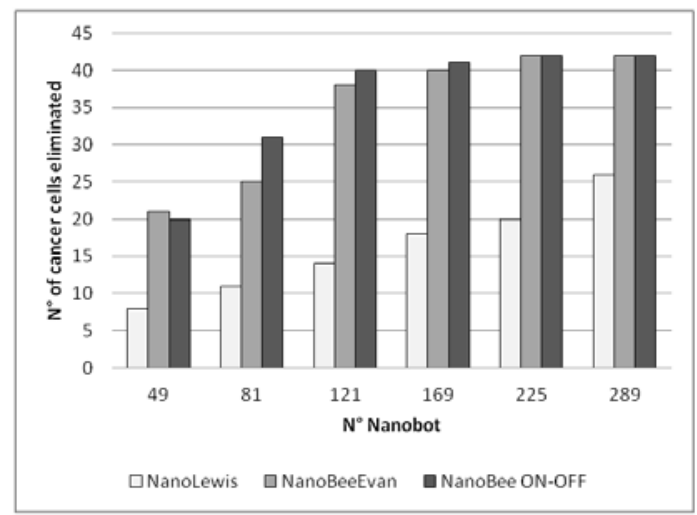

Fig. 8. Number of eliminated cancer cells in the case of "scattered groups" configuration.

In Fig. 9 we can notice how the effects of the randomness improves the behavior of the NanoBeeEvan that is very close in terms of performance to the NanoBee ON-OFF.

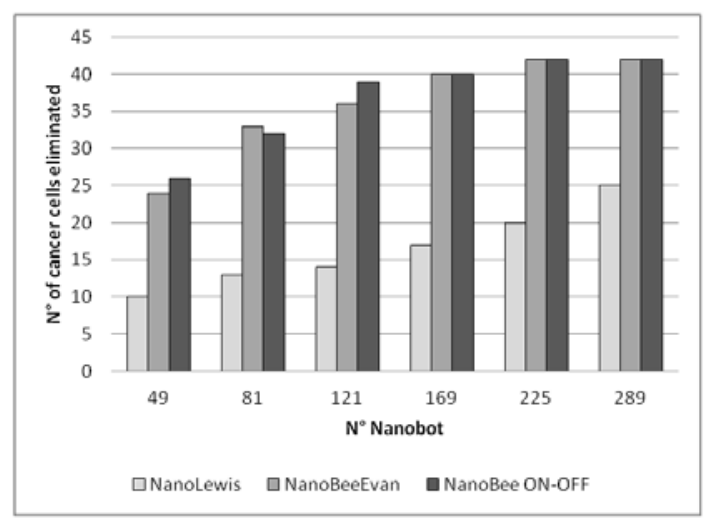

Fig. 9. Number of eliminated cancer cells in the case of "isolated" configuration.

From the analysis conducted is clear that the algorithms considered with the associated parameters in terms of size of devices, available power, etc. are feasible for nanobots operating in the specific environment as we defined (i.e. the brain). When a different environment is considered, the characteristic parameters have 
to be re-defined and the effectiveness of the technique needs to be proven. Invivo applications need a very accurate choice of available parameters, that can change based on the specific application they are considered for.

\section{Conclusions}

In this work we considered and evaluated the possibility of using and exploit a swarm of nanobots in medical applications for the treatment of endogenous diseases and through this preliminary study we showed how this is possible. Specifically, we focused on the communication and organization aspects, necessary for the control of a single nanobot through very simple rules and based only on local information exchange. We took inspiration from a previous work where the behavior of the swarms of nanobots was defined through chemical signals able to attract the devices. After an accurate evaluation of the types of communication physically feasible for in vivo communications and based on the bees behavior in the supply phase, we proposed an algorithm based on acoustic signals, NanoBee. This choice is supported from the common use of sound waves for medical applications. By assuming the possibility for a nanobot to generate a vibration when it picks up a cancer cellular tissue, we evaluated this approach and compared the algorithms via simulation by a neural simulation tool, NSL. We showed that nano-bee techniques are more effective to face cancer with different initial shape than Lewis'approach, by eliminating all the malignant cells. Communications are more efficient and nodes only need to perceive the "sound" by moving a step at each time. We showed how the proposed techniques based on the bees behaviors are effective also when the number of nanobots is smaller than that considered in the Lewis and Bekey technique.

\section{Future Works}

Concerning the future works there are many aspects that are worth to be considered in a deep way. The first one is concerning the chemical communication approach considered from Lewis and Bekey. In fact, during the simulation we were able to observe that the "barrier effect" worsens when CHEM-2 and CHEM-3 are activated. Maybe, an approach with only a single chemical substance would be more effective, and in terms of nanobot design it would be more feasible. An other important aspect is the validation of the probabilistic approach we adopted to simulate the attenuation of the signal with the square of the distance. It would be interesting to evaluate a real model of the brain and compare it with our probabilistic approach. Another important aspect to be considered is related to a more realistic simulation environment, where "obstacles" (i.e. others cells) are explicitly taken into account and the interaction of the signals is analyzed. 


\section{References}

1. Cavalcanti A., Shirinzadeh B., Zhang M., Kretly L.: Nanorobot Hardware Architecture for Medical Defense. ACM Trans. Program. Lang. Syst. vol. 8, n. 5, pp. 2932-2958, May (2008)

2. Davis M., Zuckerman J., Choi C., Seligson D., Tolcher A., Alabi C., Heidel J., Ribas A.: Nanorobots in Brain Tumor. In: International Research Journal of Pharmacy. Vol. 2, n. 2 pp.53-63, February, (2011)

3. Amato P., Masserini M., Mauri G., Cerofolini G.: Early-Stage Diagnosis of Endogenous Diseases by Swarms of Nanobots: an Applicative Scenario.Springer-Verlag Berlin, Heidelberg: ANTS'10 Proceedings of the 7-th International Conference on Swarm Intelligence, vol. 6234/2010, n. 7, pp. 408-415, September (2010)

4. Lewis A., Bekey G.: The Behavioral Self-organization of Nanorobots using Local Rules. In IEEE/RSJ IROS Conference Proceedings, pp. 1333-1338. July (1992)

5. Cavalcanti A., Hogg T., Shrinzadeth B., Liaw H. C.: Nanorobot Communication Techniques: A Comprehensive Tutorial. In IEEE International Conference of Control, Automation and Vision, ICARCV, Conference Proceedings, pp. 1-6, December (2006)

6. Hla K.H.S., Choi Y.S., Park J. S.: Mobility Enhancement in Nanorobots by using Particle Swarm Optimization. In IEEE International Conference on Computational Intelligence and Security, CIS, Conference Proceedings, pp. 35-40, December (2008)

7. Davis M., Zuckerman J., Choi C., Seligson D., Tolcher A., Alabi C., Heidel J., Ribas A.: MoEvidence of RNAi in humans from systematically administered siRNA via targeted nanoparticle. In Nature, vol. 464, n. 1064, pp. 1067-1070, April (2010)

8. Atakan B., Akan O. B.: Body Area NanoNetworks with Molecular Communications in Nanomedicine. In IEEE Communications Magazine, vol. 50, n. 1, pp. 28-34, January (2012)

9. Freitas R.: Nanomedicine, Volume I: Basic Capabilities. Landes Bioscience, (1999)

10. Weitzenfeld A., Arbib M., Alexander A.: The Neural Simulation Language. The MIT Press, (2004)

11. Dorigo M.: Optimization, Learning and Natural Algorithms. PhD Thesis, Italy, (1992)

12. Hog T., Freitas Jr R.A.: Acoustic Communication for Medical Nanorobots. In Nano Communication Networks, vol. doi:10.1016/j.nancom.2012.02.002, n. 2, pp. 1-38, January (2012)

13. Akyildiz I. F., Brunetti F., Blazquez C.: Nanoneworks: A New Communication Paradigm. In Computer Networks, vol. 52, n. 12, pp. 2260-2279, August (2011)

14. National Center for Biotechnology Information, http://www.ncbi.nlm.nih.gov

15. Loscrí V., Mannara V., Natalizio E.. Aloi G.: Efficient Acoustic Communication Techniques for Nanobots. In 7th International Conference on Body Area Networks, September 2426, 2012 Oslo, Norway (2012)

16. Stojanovic, M.: On the relationship between capacity and distance in an underwater acoustic communication channel. In Proc. of the 1st ACM Intl. Workshop on Underwater Networks (WUWNet06), pages 41-47, 2006.

17. Hogg, T., Sretavan, D. W.: Controlling tiny multi-scale robots for nerve repair. In M. Veloso and S. Kambhampati, editors, Proc. of the 20th Natl. Conf. on Arti cial Intelligence (AAAI2005), pages 1286-1291. AAAI Press, 2005 